\title{
Incidence and Reconstruction of Chronic Radiation-Induced Chest Wall Ulceration in Postoperative Breast Cancer Patients
}

\author{
Min Woo Park ${ }^{1}$ (D), Daegu Son ${ }^{1}$ (D), Sun Young Kwon² (D), Jin Hee Kim³ (D) \\ Departments of ${ }^{1}$ Plastic and Reconstructive Surgery, ${ }^{2}$ Pathology, and ${ }^{3}$ Radiation Oncology, Keimyung University School of Medicine, Daegu, Korea
}

\begin{abstract}
Background: Breast cancer patients who receive radiotherapy for postoperative tumor control are exposed to the possibility of adverse effects including osteoradionecrosis and ulceration. The purpose of this study is to explore reconstruction of ulcers according to their characteristics, and also to investigate the incidence of skin ulcers in patients who had undergone radiotherapy after modified radical mastectomy (MRM). Methods: Data of patients who underwent radiation therapy after MRM from 1979 to 2003 were collected. Demographics and radiation details were analyzed along with their reconstruction outcomes and pathologic characteristics. Effects of surgical debridement and reconstruction strategies for success were also assessed.

Results: A total of 113 patients had radiotherapy after MRM in our institution, out of which nine patients (7.96\%) developed ulcerations in the chest wall. Eleven patients underwent reconstructive surgery, including the nine patients who developed chest wall ulcerations and an additional two patients from other hospitals. Seven patients had thoracoepigastric flaps while three had latissimus dorsi musculocutaneous flaps and one had a perforator flap. The average initial wound size was $5.77 \mathrm{~cm}^{2}$, and this average was enlarged by 23.6 times after debridement. There was no reconstructive failure in any of the patients. Fibrosis, necrosis with ulceration, and dystrophic calcification were common pathological findings. Dystrophic calcification was found in seven of 11 cases. Additionally, ossification with bone formation was noted in three of seven cases with dystrophic calcification.

Conclusion: A thorough preparation of the wound bed and reconstruction with flaps appropriate for the defect can successfully treat chronic radiation-induced ulceration.
\end{abstract}

Keywords: Radiation induced abnormality; Radiotherapy; Skin ulcer; Breast cancer

\section{Introduction}

To reduce recurrence rates and improve outcomes, radiation therapy is essential for breast cancer patients who have received breast-conserving surgery. However, notwithstanding the benefits of radiation therapy, radiation-induced dermatitis develops in almost $95 \%$ of patients [1], some of whom require surgical therapy for the radiation-induced ulceration. In some cases, radiation-induced ulceration develops over 10 years after radiation [2]. Because of the ulcers' unique pathophysiology including necrosis, atrophy, fibrosis, vascular damage, carcinogenesis, and impaired cellular repair potential, healing is usually delayed and some ulcerations become chronic [3].

Various treatment modalities can be used to treat radiation-induced ulceration. Hyperbaric oxygen therapy is a conservative treatment for refractory skin ulcers after radical mastectomy and radiation therapy [4]. Tissue expansion in non-irradiated adjacent skin to cover an ulcer area nearby has also been recommended for reconstruction [5]. However, such conservative modalities are generally less effective and surgical reconstruction is required in most patients because the irradiated tissues are in

\section{Original Article}

Received: April 3, 2020

Revised: June 17, 2020

Accepted: June 18, 2020

\section{Corresponding author:}

Daegu Son, Ph.D.

Department of Plastic and Reconstructive Surgery, Keimyung University School of Medicine, 1035 Dalgubeol-daero, Dalseo-gu, Daegu 42601, Korea

Tel: +82-53-258-7815

Fax: +82-53-258-4590

E-mail: handson@dsmc.or.kr

This is an Open Access article distributed under the terms of the Creative Commons Attribution Non-Commercial License (https://creativecommons.org/licenses/by-nc/4.0/) which permits unrestricted non-commercial use, distribution, and reproduction in any medium, provided the original work is properly cited.

(c) 2020 Korean Wound Management Society 
poor condition $[3,6]$. This is because once ulceration occurs, it does not heal well, and necrotic tissue can spread much deeper and wider than what is visible. Tissue injury caused by radiation therapy is not limited to skin and soft tissues; it sometimes even damages the bones.

This study aims to explore reconstruction of ulcers of different characteristics. We also provide a summary of common characteristics of chronic radiation-induced ulceration and the essential elements required for successful reconstruction. Another purpose of this study is to investigate the incidence of skin ulcers in patients who had undergone radiotherapy after modified radical mastectomy (MRM) in our institution over a period of 24 years.

\section{Methods}

After gaining approval from the Institutional Review Board of Keimyung University Dongsan Hospital (IRB No. 2020-01028 ) and obtaining written informed consent from each patient, the incidence of ulceration was investigated in patients who received radiotherapy after MRM for breast cancer in the radiation oncology department from August 1979 to June 2003. Data was also collected retrospectively from all patients diagnosed with a chronic radiation-induced ulcer requiring reconstructive surgery by the plastic and reconstructive surgery department from May 2007 to October 2016. We looked at two separate timeframes to ensure all patients with ulcerations were included, while transferred patients were left out, because radiation-induced ulcers generally occur several years to decades after radiotherapy.

\section{Chart review}

First, data of patients were collected retrospectively from a total of 113 patients who visited the radiation oncology department for radiation therapy after MRM with breast cancer from 1979 to 2003. The data included age, laterality (left, right or both), history of diabetes, duration of radiation therapy, dose (Gy) and fraction, and additional radiation field beside the chest wall, regardless whether neoadjuvant chemotherapy was administered.

After that, more comprehensive data were collected from 11 patients who underwent reconstructive surgery. Data included demographics, body mass index, history of tobacco use, underlying diseases (diabetes, hypertension, etc.), history of radiation therapy including total dose (Gy) on each radiation field, preoperative imaging work-up and results, latent period until ulceration development after radiation therapy, wound size before and after debridement, presence of wound infection, method of reconstruction surgery, cooperation with thoracic surgery department, intraoperative findings, duration of wound healing after reconstruction surgery, results of pathological evaluation, and complications (Table 1).

\section{Reconstructive surgery}

Preoperative evaluation

Chronic radiation-induced ulcerations were often covered with thick eschar, slough, or a fistula with a small opening and purulent discharge. The skin around the ulceration was fibrous and fixed with very thin subcutaneous fat (if any), pigmented, shiny and with a visible bony outline (Fig. 1). At the same time, patients complained of impairment in movements of the shoulder and upper arm due to contraction of the axilla. The patients' underlying diseases and general conditions were checked. Computer tomography and magnetic resonance imaging were taken to confirm chronic osteomyelitis, abnormality of the bony thorax, and cancer recurrence. Since most ulcers were accompanied by infection, the presence of bacterial infection was determined by performing wound smear and deep tissue wound culture.

\section{Wound bed preparation}

In most patients, surgical debridement was performed in the operating room at the same time as the reconstructive surgery. All devitalized tissues including eschar and infected tissue and the surrounding fibrotic and calcified soft tissue were thoroughly debrided. In addition, necrotic bone was observed in most patients. Powder-like bone and sequestrum were scraped away. Where the patient suffered a fractured rib, it was completely removed in cooperation with a thoracic surgeon. Extra care was taken because parietal pleura could be exposed and hidden cavities could be uncovered (Fig. 2). Devitalized tissues and fibrous scar tissues were removed to release axillary contracture.

The excised soft tissue and bone were sent to the laboratory medicine and pathology departments for wound culture and histologic evaluation, respectively. Pathological evaluation was done by a single blinded pathologist. Wound infection was controlled using intravenous antibiotics with surgical debridement. For wound dressing, povidone iodine solution was most commonly used for wet-to-dry dressing. 
Table 1. Reconstruction patient demographics and summary of treatment outcomes and complications

\begin{tabular}{|c|c|c|c|c|c|c|c|c|c|c|}
\hline \multirow{2}{*}{$\begin{array}{l}\text { Patient } \\
\text { №. }\end{array}$} & \multirow[b]{2}{*}{ Sex } & \multirow{2}{*}{$\begin{array}{l}\text { Age } \\
\text { (yr) }\end{array}$} & \multirow{2}{*}{$\begin{array}{c}\mathrm{BMI} \\
\left(\mathrm{kg} / \mathrm{m}^{2}\right)\end{array}$} & \multirow{2}{*}{$\begin{array}{l}\text { Underlying } \\
\text { diseases }\end{array}$} & \multirow{2}{*}{$\begin{array}{l}\text { Time of ulcer } \\
\text { development } \\
\text { after RT (yr) }\end{array}$} & \multicolumn{2}{|c|}{ Wound size $\left(\mathrm{cm}^{2}\right)$} & \multirow{2}{*}{$\begin{array}{l}\text { Reconstructive } \\
\text { surgery }\end{array}$} & \multirow[b]{2}{*}{ Complications } & \multirow{2}{*}{$\begin{array}{l}\text { Follow-up } \\
\text { (mon) }\end{array}$} \\
\hline & & & & & & Initial & Final & & & \\
\hline $1^{\text {a) }}$ & $\mathrm{F}$ & 73 & 28.5 & HT, DM & 36.0 & 1.0 & 9.0 & $\begin{array}{l}2 \text { Perforator } \\
\text { flaps }\end{array}$ & $\begin{array}{l}\text { Skin graft loss, } \\
\text { wound dehiscence }\end{array}$ & 14 \\
\hline 2 & $\mathrm{~F}$ & 58 & 24.1 & - & 26.0 & 1.0 & 216.0 & LD & - & 12 \\
\hline 3 & $\mathrm{~F}$ & 64 & 26.1 & HT & 23.0 & 1.0 & 180.0 & TE & - & 12 \\
\hline 4 & M & 80 & 26.4 & AP, BPH, COPD, DM, HT & 5.0 & 0.3 & 225.0 & TE & Hematoma & 10 \\
\hline 5 & $\mathrm{~F}$ & 58 & 29.4 & - & 8.2 & 1.0 & 150.0 & TE & Partial flap necrosis & 18 \\
\hline 6 & $\mathrm{~F}$ & 77 & 29.9 & $\begin{array}{l}\text { HT, OA, } \\
\text { hyperthyroidism, GE }\end{array}$ & 16.2 & 2.3 & 52.5 & LD & - & 2 \\
\hline 7 & $\mathrm{~F}$ & 58 & 24.2 & DM & 12.0 & 20.0 & 225.0 & TE & - & 12 \\
\hline 8 & $\mathrm{~F}$ & 49 & 24.1 & - & 5.3 & 25.0 & 144.0 & TE & - & 19 \\
\hline 9 & $\mathrm{~F}$ & 68 & 21.2 & - & 13.0 & 9.0 & 100.0 & TE & $\begin{array}{l}\text { Minimal tissue } \\
\text { necrosis }\end{array}$ & 12 \\
\hline $10^{\text {a) }}$ & $\mathrm{F}$ & 73 & 23.6 & $\begin{array}{l}\text { HT, DM, } \\
\text { hyperlipidemia }\end{array}$ & 21.0 & 2.0 & 100.0 & TE & - & 11 \\
\hline 11 & $\mathrm{~F}$ & 70 & 25.1 & - & 11.0 & 1.0 & 98.0 & LD & - & 20 \\
\hline Average & & $6.20 \pm 9.59$ & $25.69 \pm 2.69$ & & $16.06 \pm 9.59$ & $5.77 \pm 8.68$ & $136.32 \pm 71.81$ & - & - & $12.90 \pm 4.99$ \\
\hline
\end{tabular}

BMI, body mass index; RT, radiation therapy; F, female; M, male; HT, hypertension; DM, diabetes mellitus; LD, Latissimus dorsi flap; TE, thoracoepigastric flap; AP, angina pectoris; $\mathrm{BPH}$, benign prostate hyperplasia; $\mathrm{COPD}$, chronic obstructive lung disease; $\mathrm{OA}$, osteoarthritis; $\mathrm{GE}$, gastroesophageal reflux.

a)Patients referred from outside.
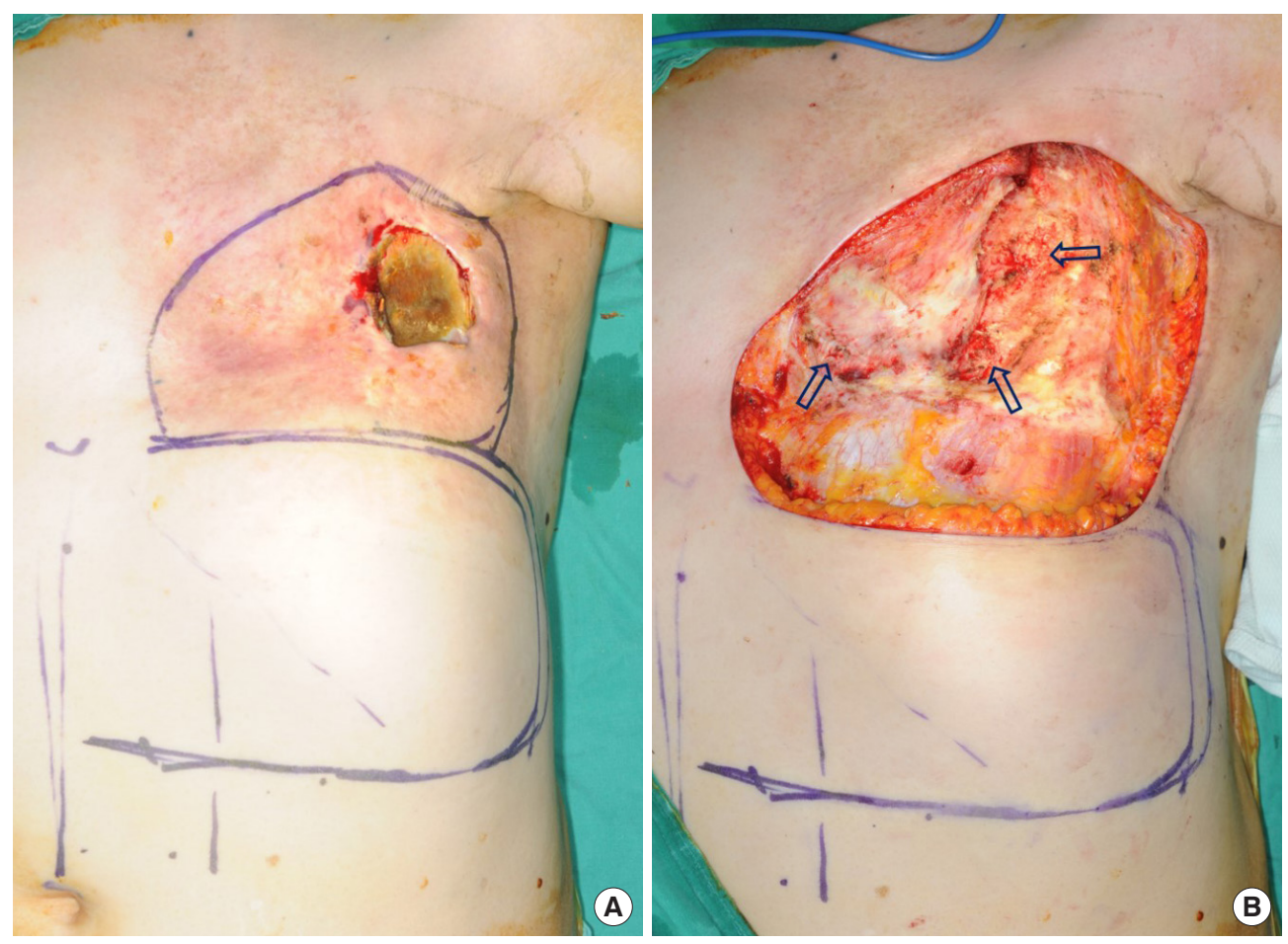

Fig. 1. Typical wound of radiation-induced chronic chest wall ulceration. (A) Before debridement, the wound is covered with thick eschar, multiple calcifications on the left fourth and fifth ribs and parietal pleura. (B) True defects are observed after complete debridement, including the entire eschar, necrotic bone (arrows) with partial resection of the fourth rib, and fibrotic tissues. 

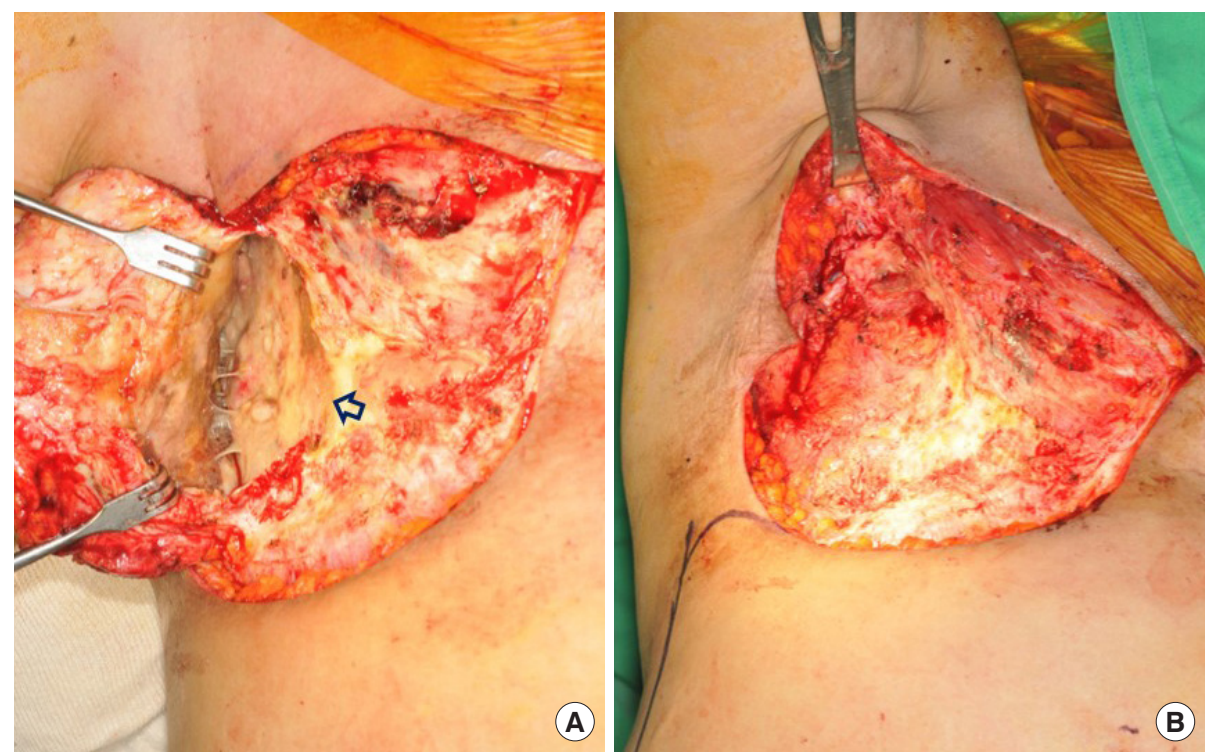

Fig. 2. Encapsulated hidden cavity found under the fibrotic skin. (A) A cavity (indicated with arrow) surrounded by a yellow and dirty membrane. (B) After debridement and release of a contracture by removing all devitalized and fibrotic scar tissues at the chest and axilla.
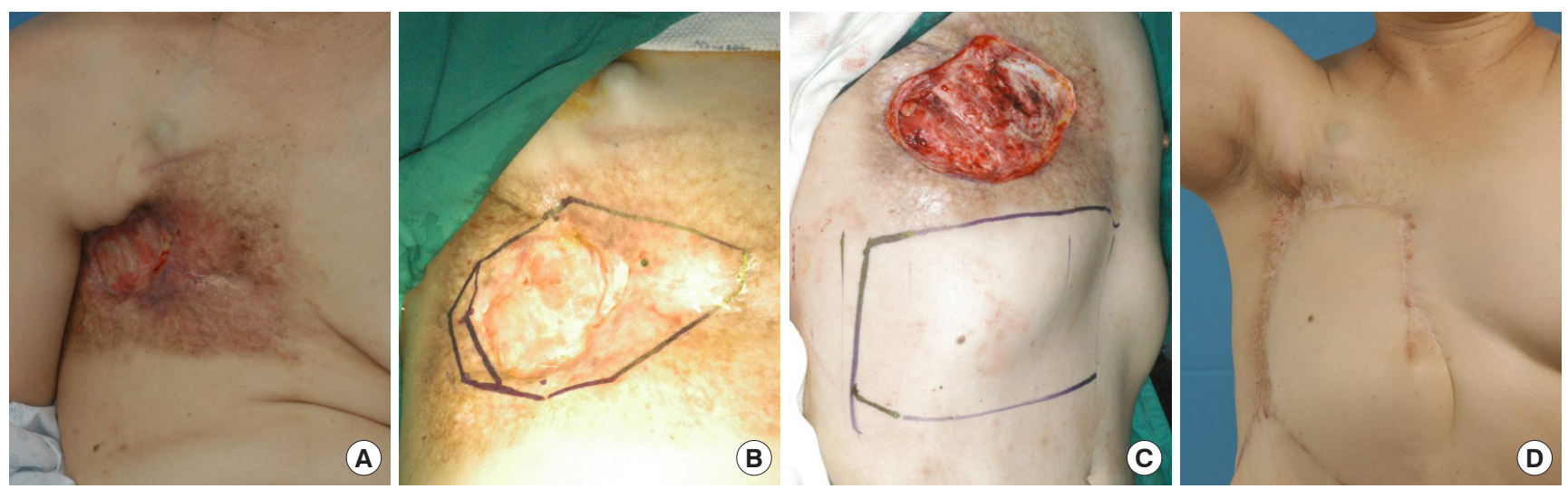

Fig. 3. Reconstruction with a thoracoepigastric flap. (A) This photograph shows the typical features of radiation-induced chronic ulcers before surgery. (B) Preoperative resection range is designed to include ulcers and surrounding unstable tissue. (C) The base of the flap is based on perforating vessels of the rectus muscle. The flap extends laterally to the midaxillary line and inferiorly to the middle of the xiphoid process and umbilicus. (D) At 19 months after surgery, there was no complication, and complete improvement of the axillary contracture was observed.

\section{Flap selection and reconstruction}

Complete and thorough debridement and contracture release were performed to reveal real defects. The choice of flaps was based on the size of the real defect, the extent of tissue defects, and the patient's general condition. In the present study, thoracoepigastric (TE) flap and latissimus dorsi musculocutaneous (LD) flap were used for most of the cases. Although TE flap has the advantage that it can be elevated quickly and adjacent to the ulcer, it is not suitable for deep wounds because it is a cutaneous flap that does not include muscles $[7,8]$. The TE flap was used when the patient's general condition was poor, be- cause such a flap could be elevated and transferred quickly (Fig. 3).

Meanwhile, because the LD flap has good vascularity and muscles, it can be used to reconstruct large and deep defects with poor vascular bed (Fig. 4).

\section{Statistical analysis}

Microsoft Excel (Microsoft Corp., Redmond, WA, USA) was used to calculate the standard deviation. Statistically significant differences between radiation field and total dose were determined by $\mathrm{T}$ or chi-square test and Fisher exact test. Val- 

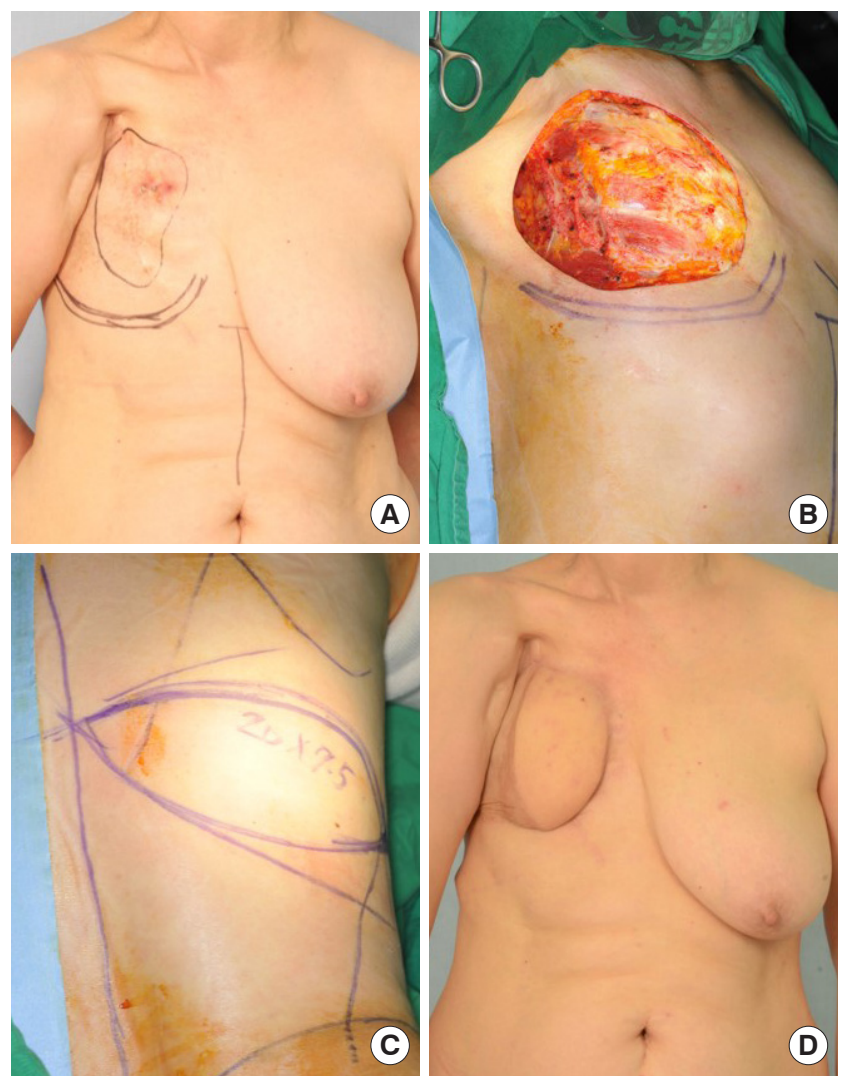

Fig. 4. Reconstruction with a latissimus dorsi (LD) flap. (A) Preoperative design of extent of debridement. (B) After complete excision of all devitalized tissues including fibrotic and calcified soft tissues and necrotic bone. (C) Design of LD flap. (D) At 13 months after surgery.

ues of $\mathrm{P}<0.05$ were considered statistically significant when one comparison was being made.

\section{Results}

A total of 113 patients visited the radio-oncology department for radiation after MRM during the study period. Among them, nine patients (7.96\%) developed radiation-induced ulceration requiring surgical intervention. The patient's mean age at the time of radiation therapy was 47 years old for the non-ulcer group and 48.8 years old for the ulcer group. All patients had radiation therapy in the 2 -dimensional method, and the average duration of radiation therapy was 50.57 days for the non-ulcer group, and 47 days for the ulcer group. Among 97 non-ulcer patients who received additional field radiation besides the chest wall, 57 (54.8\%) had additional supraclavicular lymph node (SCL) and axillary lymph node (ALN) radiation and $18(17.3 \%)$ had only additional SCL radiation. In the
Table 2. Demographics of radiation patients with ulcers versus patients without ulcers

\begin{tabular}{lccc}
\hline Characteristics & $\begin{array}{c}\text { Ulcer } \\
\text { group }\end{array}$ & $\begin{array}{c}\text { Non-ulcer } \\
\text { group }\end{array}$ & P-value \\
\hline No. of patients & $9(7.96)$ & $104(92.03)$ & \\
Mean age (yr) & $48.80 \pm 11.62$ & $47.00 \pm 10.03$ & 0.651 \\
Laterality & & & \\
Right & $5(4.42)$ & $50(44.24)$ & \\
Left & $4(3.53)$ & $53(46.90)$ & \\
Both & 0 & $1(0.88)$ & \\
Neoadjuvant chemotherapy & 0 & $10(8.84)$ & 0.33 \\
Additional radiation field & & & \\
SCL & 0 & $18(17.30)$ & 0.174 \\
IMN & 0 & $3(2.88)$ & 0.606 \\
Axilla & 0 & $4(3.84)$ & 0.549 \\
SCL+IMN & 0 & $6(5.77)$ & 0.459 \\
SCL+ALN & $6(66.66)$ & $57(54.80)$ & 0.492 \\
IMN+ALN & $2(22.22)$ & $3(2.88)$ & $0.047^{\text {a) }}$ \\
SCL+IMN+ALN & 0 & $6(5.77)$ & 0.459 \\
Duration of radiation therapy (day) & $47.00 \pm 40.00$ & $50.57 \pm 13.83$ & 0.098 \\
Average radiation dose (Gy) & & & \\
WB/CW (9:104) & & & \\
SCL (6:87) & $52.95 \pm 60.00$ & $50.45 \pm 3.51$ & 0.217 \\
IMN (2:18) & $49.33 \pm 20.00$ & $50.10 \pm 4.87$ & 0.381 \\
ALN (8:70) & $50.20 \pm 0.00$ & $48.60 \pm 7.74$ & 0.779 \\
\hline b) & $49.55 \pm 10.00$ & $49.83 \pm 2.03$ & 0.705 \\
\hline
\end{tabular}

Values are presented as number (\%) or mean \pm SD.

SCL, supraclavicular lymph node; IMN, internal mammary lymph node; ALN, axillary lymph node; WB/CW, whole breast/chest wall.

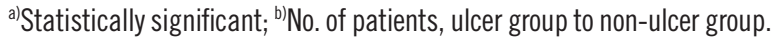

ulcer group, eight patients received additional field radiation therapy. There were significantly more patients who were subject to both additional internal mammary lymph node (IMN) and ALN radiation in the ulcer group $(\mathrm{P}=0.047)$. The mean total doses of each radiation field are demonstrated on Table 2.

Two patients who visited the plastic reconstructive surgery department previously had radiation therapy at another medical center, making the total number of patients who had reconstruction surgery at our department 11 (1 male and $10 \mathrm{fe}$ males). Their mean age was 66.2 years at the time of surgery. The average time of ulceration development after radiation therapy was 16 years. Four out of 11 patients showed infected wounds. Three patients had staphylococcal infections, while two had pseudomonas infections. One patient had multiple overlapping infections.

Seven patients had reconstruction using TE flaps while 

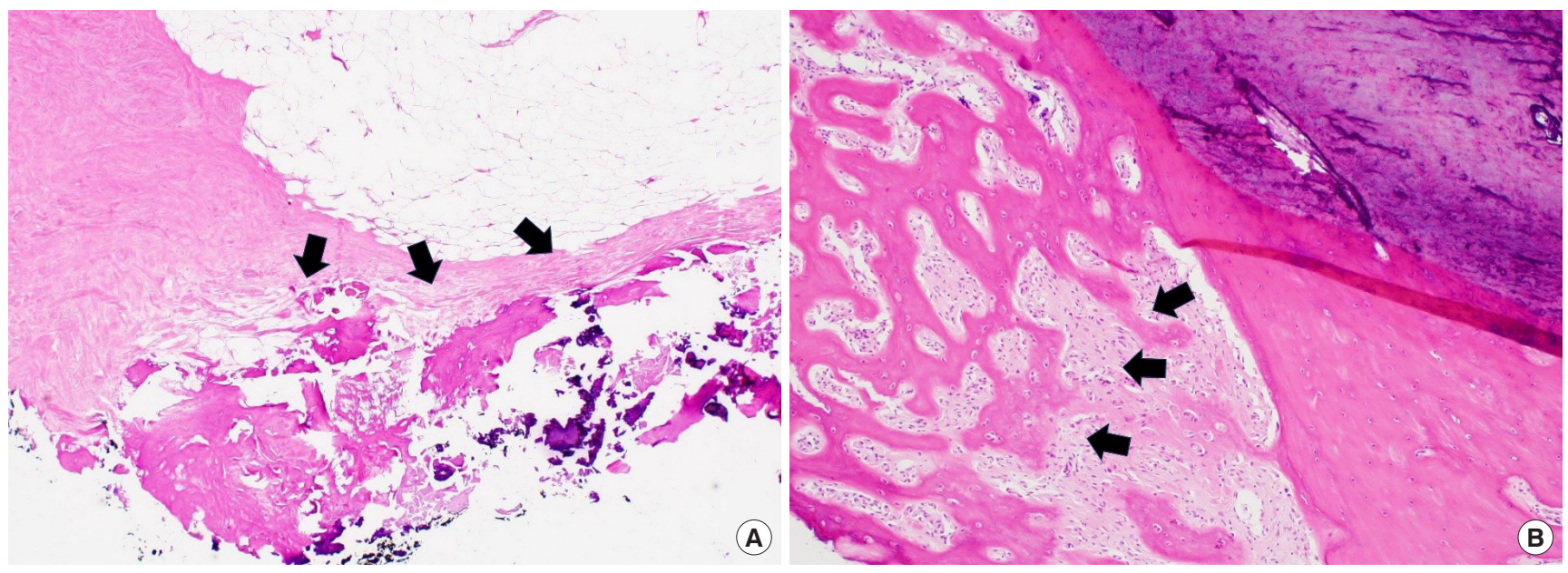

Fig. 5. Histologic features of radiation-induced chronic ulcerative tissues. (A) Dystrophic calcification (indicated with arrows) within fibrosis $(H \& E, \times 40)$. (B) Dystrophic calcification progresses to ossification (H\&E, $\times 400)$.

three had LD flaps for reconstruction. One patient underwent reconstruction using a perforator flap. Cooperation with a thoracic surgeon for necrotic rib resection was required in three cases. The average initial wound size was $5.77 \mathrm{~cm}^{2}$, enlarged 23.6 times after debridement $\left(136.32 \mathrm{~cm}^{2}\right)$. Patients were discharged at an average of 11.09 days after surgery. The average follow-up period for healing was 12.9 months. Results are summarized in Table 1.

Histologic features of radiation-induced chronic ulcerative tissues show typically thinned epidermis with loss of skin adnexa and diffuse fibrosis and necrosis within deep dermis and subcutaneous tissue. All cases included in the study presented diffuse fibrosis and little vascularity, confirmed by Masson trichrome and hematoxylin and eosin staining. Additionally, variable degrees of acute and chronic inflammation were noted in all the cases. Dystrophic calcification within markedly fibrotic change was found in seven cases (Fig. 5A). In particular, focal ossification with bone formation was observed in three of the seven cases of dystrophic calcification (Fig. 5B). Fat necrosis was seen in five cases and extensive surface ulceration was present in three cases.

Revision surgery was performed in two cases. In the first case, reconstruction with a TE flap was performed; but after one month, a 3-mm sized ulceration with fistula formed on the upper margin of the flap. During the operation, erosion of the fourth rib, not found in the previous surgery, was observed. Therefore, we debrided and partially resected the rib before primary closure. In the second case, a superficial skin necrosis was treated with a skin graft.

\section{Discussion}

The foremost step in evaluating chronic wounds after radiation therapy in breast cancer patients is to rule out the recurrence of cancer $[9,10]$. Yi et al. [9] have reported that radiation-induced malignancies such as invasive ductal carcinoma, lymphoma, and angiosarcomas can develop as late complications with a latency of more than 5 years. Fortunately, there was no cancer recurrence in our patients with radiation-induced chronic ulcers.

Long-term sequelae of radiation include necrosis, atrophy, fibrosis, vascular damage, and carcinogenesis [11,12]. Results of the present study were not different from those of previous studies. However, one caveat is that these histological findings are not limited to ulcers. They are also found under the entire fibrotic scar tissue treated with radiation. In our study, dystrophic calcification within markedly fibrotic change was found in $64 \%$ of ulcer patients. The most common causes of dystrophic calcification are soft tissue trauma and inflammation. As the disease progresses, calcified tissues begin to organize and ossify over the course of months into lamellar bone [13]. These heterotopic calcifications and ossifications suggest that these lesions are end-stage damage following high-dose radiotherapy [14], proving that these tissues should be completely excised and covered with highly vascularized axial pattern flaps.

Microvascular injury and defective collagen deposition are major reasons in the pathophysiology of radiation ulcers that result in poor wound healing. In the present study, histological examination showed little vascularity in ulcer and fibrotic tissues, suggesting that healing is very difficult even if the ulcer is 
small. This is typical of irradiated tissues that are not likely to spontaneously heal $[15,16]$, making simple procedures like local wound care and skin grafts insufficient for wound healing $[17,18]$. Good results can only be achieved by reconstruction surgery using highly vascularized flaps. This finding is supported by our biopsy data. Intraoperative findings also indicated that most ulcers had fibrosis and poor blood supply that require skin flaps or muscle flaps with a good supply of blood.

There is no general consensus on dimensions for debridement of radiation-induced ulcers. In our data, the initial size of the external wound was $5.77 \mathrm{~cm}^{2}$ on average. After complete debridement, the average size was $136.32 \mathrm{~cm}^{2}$, which was an enlargement of around 23.6 times. Surgeons should keep in mind that surgical debridement is to be performed until marginal tissues are assured healthy enough to heal spontaneously.

There was no significant difference in total radiation dose, or history of diabetes between the ulcer group and non-ulcer group, except the subgroup that received additional radiation to the IMN and ALN at the same time. However, clinically, eight out of nine ulcer patients received additional field radiation therapy and the most severe part of their ulcers appeared to be in the junction between the two radiation fields of the chest wall and the additional area. In this study, most patients had radiation therapy in 2-dimensional techniques, as opposed to the intensity-modulated radiation therapy commonly used these days. Due to a unique radiation delivery mechanism of photons and electrons, irradiation in the junction area may be greater than planned. Even if the moving-field technique is applied, irradiation might have been disproportionately high in the junction of the two fields, possibly causing radiation-induced ulceration. Analysis of patients who received additional IMN and ALN radiation found them to have statistically significantly higher occurrence of ulceration. However, the overall number of patients in the ulcer group was small, which may result in low statistical power. Further study of junctions in irradiation and ulceration development is required.

The shortcoming of our research was that there were only a small number of cases, and not much diversity. All patients in this study were group 2 (moderate type) according to the classification of Ma et al. [19]. Several studies have noted that reconstruction of irradiated chest ulcers must provide pleural and structural continuity to support the breathing mechanism, such as using a synthetic mesh or rib graft to reinforce the chest wall [20-22]. The more severe the ulceration is, the more cautious and multidisciplinary an approach is required.

In conclusion, reconstruction of radiation-induced chronic ulcers should be based on awareness that the true defect is much larger than the apparent defect when there is widespread damage of tissue. A thorough wound bed preparation, and reconstruction with a flap that is appropriate for the true defect can contribute to successful treatment of radiation-induced chronic ulcerations.

\section{Conflict of interest}

No potential conflicts of interest relevant to this article are reported.

\section{ORCID iDs}

Min Woo Park https://orcid.org/0000-0003-2156-1053

Daegu Son

Sun Young Kwon

Jin Hee Kim https://orcid.org/0000-0002-4653-1048 https://orcid.org/0000-0002-8410-0185 https://orcid.org/0000-0001-8608-9250

\section{References}

1. Hickok JT, Morrow GR, Roscoe JA, et al. Occurrence, severity, and longitudinal course of twelve common symptoms in 1129 consecutive patients during radiotherapy for cancer. J Pain Symptom Manage 2005;30:433-42.

2. Schindl A, Schindl M, Schind L. Phototherapy with low intensity laser irradiation for a chronic radiation ulcer in a patient with lupus erythematosus and diabetes mellitus. $\mathrm{Br}$ J Dermatol 1997;137:840-1.

3. Zhou Y, Zhang Y. Single- versus 2-stage reconstruction for chronic post-radiation chest wall ulcer: a 10-year retrospective study of chronic radiation-induced ulcers. Medicine (Baltimore) 2019;98:e14567.

4. Enomoto M, Yagishita K, Okuma K, et al. Hyperbaric oxygen therapy for a refractory skin ulcer after radical mastectomy and radiation therapy: a case report. J Med Case Rep 2017;11:5.

5. MacMillan RW, Arias JD, Stayman JW 3rd. Management of radiation necrosis of the chest wall following mastectomy: a new treatment option. Plast Reconstr Surg 1986;77:832-5.

6. Makboul M, Salama Ayyad MA. Is myocutaneous flap alone sufficient for reconstruction of chest wall osteoradionecrosis? Interact Cardiovasc Thorac Surg 2012;15:447-51.

7. Davis WM, McCraw JB, Carraway JH. Use of a direct, transverse, thoracoabdominal flap to close difficult wounds of the thorax and upper extremity. Plast Reconstr Surg 1977; 
60:526-33.

8. Baroudi R, Pinotti JA, Keppke EM. A transverse thoracoabdominal skin flap for closure after radical mastectomy. Plast Reconstr Surg 1978;61:547-54.

9. Yi A, Kim HH, Shin HJ, et al. Radiation-induced complications after breast cancer radiation therapy: a pictorial review of multimodality imaging findings. Korean J Radiol 2009;10:496-507.

10. Granick MS, Larson DL, Solomon MP. Radiation-related wounds of the chest wall. Clin Plast Surg 1993;20:559-71.

11. Dormand EL, Banwell PE, Goodacre TE. Radiotherapy and wound healing. Int Wound J 2005;2:112-27.

12. Baker DG, Krochak RJ. The response of the microvascular system to radiation: a review. Cancer Invest 1989;7:287-94.

13. Mujtaba B, Taher A, Fiala MJ, et al. Heterotopic ossification: radiological and pathological review. Radiol Oncol 2019;53:275-84.

14. Carl UM, Hartmann KA. Heterotopic calcification as a late radiation effect: report of 15 cases. Br J Radiol 2002;75:4603.
15. Robinson DW. Surgical problems in the excision and repair of radiated tissue. Plast Reconstr Surg 1975;55:41-9.

16. Strawberry CW, Jacobs JS, McCraw JB. Reconstruction for cervical irradiation ulcers with myocutaneous flaps. Head Neck Surg 1984;6:836-41.

17. Rudolph R. Complications of surgery for radiotherapy skin damage. Plast Reconstr Surg 1982;70:179-85.

18. Shack RB. Management of radiation ulcers. South Med J 1982;75:1462-6.

19. Ma X, Jin Z, Li G, et al. Classification of chronic radiationinduced ulcers in the chest wall after surgery in breast cancers. Radiat Oncol 2017;12:135.

20. Maruyama Y, Onishi K, Iwahira Y. Reconstructing chest walls with vertical abdominal fasciocutaneous flaps. Scand J Plast Reconstr Surg 1986;20:79-83.

21. Fujioka M. Surgical reconstruction of radiation injuries. Adv Wound Care (New Rochelle) 2014;3:25-37.

22. Raz DJ, Clancy SL, Erhunmwunsee LJ. Surgical management of the radiated chest wall and its complications. Thorac Surg Clin 2017;27:171-9. 